\title{
Le questionnaire médical en odontostomatologie : nécessité, applications, obligations
}

\section{RÉSUMÉ}

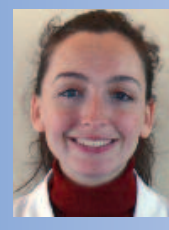

Caroline GORIN

Attachée de consultation à I'université Paris Descartes,

1 rue Maurice-Arnoux,

92120 Montrouge.

Sophie-Myriam DRIDI

Maître de conférence

à l'université Paris Descartes,

1 rue Maurice-Arnoux,

92120 Montrouge.

Claude-Bernard WIERZBA

Maître de conférence

à l'université Paris Descartes,

1 rue Maurice-Arnoux,

92120 Montrouge.

Anne-Laure EJEIL

Maître de conférence

à l'université Paris Descartes,

1 rue Maurice-Arnoux,

92120 Montrouge.
Un grand merci au Docteur Jean Meyer pour son aide précieuse et amicale.

L'enquête médicale, souvent banalisée, est un outil de travail indispensable dans notre pratique quotidienne. Elle se présente sous la forme d'un questionnaire écrit, daté et signé par le patient, complété d'un entretien oral avec le praticien qui peut alors apprécier l'état de santé général du patient, son suivi médical, ainsi que les médications passées, en cours ou à venir.

L'odontologiste peut donc planifier correctement les différentes étapes du plan de traitement et prendre les décisions thérapeutiques qui s'imposent, avec toutes les précautions médicales et techniques nécessaires.

- enquête médicale

- questionnaire médical

- recommandations

- patients à risque
AOS 2011;253:19-37

DOI: 10.1051/aos/2011103

(C) AEOS / EDP Sciences 


\section{Introduction}

$>$

En pratique quotidienne, I'odontologiste, réalise des actes très diversifiés. Beaucoup d'entre eux, considérés comme banaux, peuvent néanmoins être invasifs et nécessiter des précautions de prise en charge. Aussi, quelle que soit la nature de l'acte endodontie, parodontie, chirurgie buccale, implantologie -, le praticien se doit de connaître l'état de santé général de son patient afin d'éviter certains écueils pouvant s'avérer néfastes voire fatals dans certaines situations exceptionnelles.

À cet effet, l'enquête médicale (EM) représente un outil de travail indispensable à condition que le praticien sache le décoder et en retirer l'essentiel.

Deux notions sont à considérer. L'EM représente non seulement un outil médical mais également un outil juridique médico-légal important.

L'outil médical permet de déterminer précisément la capacité des patients à recevoir des soins en fonction des risques médicaux suspectés ou notifiés, du profil psychologique de la personne et de son attitude plus ou moins coopérative. La façon dont est rempli le questionnaire peut également mettre en évidence certains traits de caractère de l'individu : nervosité, anxiété, agressivité, passivité, mélancolie... L'entretien oral permet d'approfondir les réponses consignées dans le dossier et de juger de l'état psychologique ou d'anxiété dans lequel se trouve le patient. Le carnet de santé peut être demandé en complément. Le praticien peut aisément contacter le médecin traitant si des informations supplémentaires sont nécessaires : que ce soit au sujet de l'état de santé général, de la sévérité de la pathologie, du traitement, et des examens biologiques complémentaires.

L'outil juridique repose sur l'établissement d'un document écrit complété d'un entretien oral. Le document écrit doit être simple, clair, compréhensible, complet et révéler l'état civil du patient, son état de santé actuel, les traitements chirurgicaux antérieurs ou à venir, ainsi que les coordonnées complètes du médecin traitant (tableau I). Ce même document doit être remis à jour régulièrement, daté et signé par le patient. La signature engage la responsabilité du patient quant aux informations mentionnées et l'oblige ainsi à tout déclarer. En cas d'accident grave et imprévu, le praticien peut prouver qu'il a bien respecté ses obligations de moyens.

Ainsi une EM conduite de façon efficace apporte souvent les réponses aux questions suivantes :

- le patient peut-il être traité dans un cabinet en ville ou nécessite-il une prise en charge en milieu hospitalier?

- quel type de molécule anesthésique peut-on utiliser? Quelle technique ? Avec ou sans vasoconstricteur?

- le patient présente-t-il un risque hémorragique ? Un risque infectieux ? Un risque de nécrose osseuse ? Un facteur de risque de cancer des voies aérodigestives supérieures (VADS) ?

À partir des données recueillies, l'omnipraticien est plus à même de planifier une attitude thérapeutique raisonnée et appropriée. 


\section{Tableau I Exemple type de questionnaire médical écrit.}

Madame, Mademoiselle, Monsieur,

La connaissance de votre état de santé nous conduit à adapter notre attitude thérapeutique à chaque cas particulier. C'est pourquoi nous vous remercions de l'attention que vous porterez à ce document en le remplissant. Le questionnaire est confidentiel et soumis au secret professionnel.

- Médecin traitant : Nom

Adresse

- Troubles cardio-vasculaires (date du début de l'affection et médicaments pris à cet effet) :

Hypertension artérielle Hypotension artérielle

Angine de poitrine Embolie pulmonaire

Phlébite Souffle cardiaque Valvuloplastie

Cardiopathie Transplants

- Troubles gastro-intestinaux :

Ulcère gastro-duodénal

Hépatites virales

Régurgitation acide Cirrhose hépatique

Autres

- Troubles rénaux :

Dialyse Insuffisance rénale chronique

Transplants rénaux Autres.

- Troubles allergiques :

Anesthésiques locaux

Autres

Médicaments

Latex

- Troubles respiratoires:

Bronchite chronique

Asthme

Autres

\section{- Troubles hématologiques :}

Saignements anormaux après intervention dentaire Hémophilie Anémie

Ecchymose .... Transfusion imprévue .. Autres

\section{- Troubles ostéo-articulaires:}

Arthrose

RAA

Prothèse articulaire

Autres

- Troubles neurologiques :

Épilepsie Anxiété Troubles du sommeil

Dépression nerveuse

Autres

- Troubles endocriniens :

Ménopause

Diabète

Hyper ou hypothyroïdie

Autres

- Grossesse

Allaitement

- Ostéoporose

- Séropositivité HIV

SIDA

Virémie

Taux CD4

- Intervention chirurgicale

- Radiothérapie

- Chimiothérapie

- Biphosphonate

Champs d'irradiation

Dose reçue

- Consommation tabac d'alcool

Voie intraveineuse ou orale Spasmophilie

Autres drogues

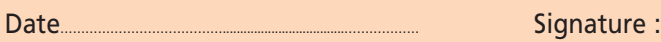




\section{Hôpital ou cabinet en ville?}

L'EM bien dirigée permet de dépister l'existence d'une pathologie systémique sévère, non stabilisée malgré une thérapie, non contrôlée ou non diagnostiquée. Pour ces situations, il est recommandé de contacter le médecin traitant par courrier pour une évaluation médicale complète et pour discuter de la possibilité de soigner le patient en cabinet de ville.

Plusieurs points doivent être éclaircis :

- le passé médical (les hospitalisations multiples révélant des problèmes hémostatiques, cardiaques, infectieux...) donne des indications sur l'état de santé général ;

- le type de pathologie et les complications associées (par exemple un patient diabétique insulinodépendant non équilibré a un potentiel de cicatrisation diminué) ;

- la persistance de symptômes malgré un traitement approprié (par exemple dyspnée au repos malgré un traitement pour une insuffisance cardiaque sévère) ;

- la date exacte d'apparition de la pathologie (par exemple un infarctus dans les six mois précédant le rendez-vous interdit les soins urgents en ville à cause de la vulnérabilité du myocarde) ;

- la fréquence des symptômes (par exemple, crise d'épilepsie) ;

- la nature du traitement et la posologie exacte de la médication en cours ;

- les résultats des tests biologiques (par exemple taux de lymphocytes T CD4 et virémie chez les patients $\mathrm{VIH}+$ ) ;

- le niveau de contrôle effectué par le médecin traitant la (ou les) pathologie(s).

Si le médecin traitant confirme l'impossibilité de prendre en charge le patient en dehors d'un cadre spécifique, il faut le diriger vers un service hospitalier et ne réaliser en ville que les actes non invasifs, tels que I'observation clinique, l'enseignement à l'hygiène orale (EHO), les conseils alimentaires et la radiographie. En situation d'urgence, la douleur et l'infection pourront être jugulées par des médications n'interférant pas avec les pathologies et les médications en cours.

\section{Anesthésiques locaux :}

quelles molécules?

\section{Avec ou sans vasoconstricteurs? Quelles techniques?}

L'EM doit mettre à jour :

- les incidents apparus après une injection antérieure d'une solution d'anesthésique local ;

- le type d'anesthésique incriminé (à fonction amine ou ester, avec ou sans vasoconstricteur : épinéphrine ou norépinéphrine) (tableau II) ; - les pathologies générales ou les médications en cours qui imposent de respecter certaines 
précautions d'usage ou qui contre-indiquent telle ou telle molécule.

\section{Les incidents}

L'EM doit permettre de préciser la nature exacte de la réaction rapportée par le patient.

Trois situations peuvent être observées [1].

\section{$>$ Les accidents mineurs}

Ce sont les plus fréquemment rencontrés. Provoqués surtout par l'anxiété, la fatigue et l'émotion, ils se manifestent le plus souvent par un malaise vagal.

L'entretien oral est d'autant plus déterminant que la même symptomatologie survient en général à l'occasion de prise de sang ou d'émotions vives.

\section{> Les accidents majeurs}

Plus rares, ils apparaissent en cas de surdosage. Ils peuvent être immédiats (injection intravas- culaire malencontreuse), ou plus tardifs, causés par l'injection d'une trop grande quantité d'anesthésique.

Ils se manifestent au niveau du système nerveux par une agitation, une convulsion, un trouble de la conscience et au pire une dépression du système nerveux central (SNC); au niveau cardiovasculaire, par une chute de la pression artérielle, des troubles du rythme pouvant même aboutir à un arrêt cardiaque.

\section{$>$ Les accidents allergiques}

Exceptionnels, ils peuvent être provoqués par les quatre constituants des solutions anesthésiantes: I'anesthésique à fonction amide ou ester, l'antioxydant, le conservateur, les vasoconstricteurs.

On parle d'allergie quand le patient rapporte des signes d'urticaire (fig. 1), de tuméfaction, de rash cutané, de crise d'asthme, d'œdème de Quincke ou de choc anaphylactique, syndrome de Lyell ou de Stevens-Johnson.

\begin{tabular}{|c|c|c|}
\hline & Dénomination internationale commune & Contre-indications \\
\hline $\begin{array}{l}\text { Anesthésiques } \\
\text { à fonction ester }\end{array}$ & Procaïne & $\begin{array}{l}\text { Déficit en cholinestérase } \\
\text { Allergie (asthme, rhume des foins, urticaire...) } \\
\text { Hypersensibilité aux anesthésiques locaux } \\
\text { ou à l'un des composants } \\
\text { Épilepsie } \\
\text { Hypotension } \\
\text { Précaution : grossesse }\end{array}$ \\
\hline $\begin{array}{l}\text { Anesthésiques } \\
\text { à fonction amide }\end{array}$ & $\begin{array}{l}\text { Lidocaïne } \\
\text { Bupivacaïne } \\
\text { Articaïne } \\
\text { Prilocaïne } \\
\text { Aptocaïne }\end{array}$ & $\begin{array}{l}\text { Hypersensibilité aux anesthésiques locaux } \\
\text { ou à l'un des composants } \\
\text { Porphyrie (sauf Aptocaïne) } \\
\text { Épilepsie non contrôlée par le traitement } \\
\text { Troubles de la conduction auriculoventriculaire } \\
\text { sévère non appareillés } \\
\text { Antécédent d'hyperthermie maligne } \\
\text { Pathologies cardiaques sévères, } \\
\text { troubles du rythme, infarctus de moins de } 6 \text { mois, } \\
\text { hyperthyroïdie pour les formes avec adrénaline } \\
\text { Précaution : insuffisance hépatique sévère avec les } \\
\text { bêtabloquants qui augmentent leurs effets indésirables }\end{array}$ \\
\hline
\end{tabular}




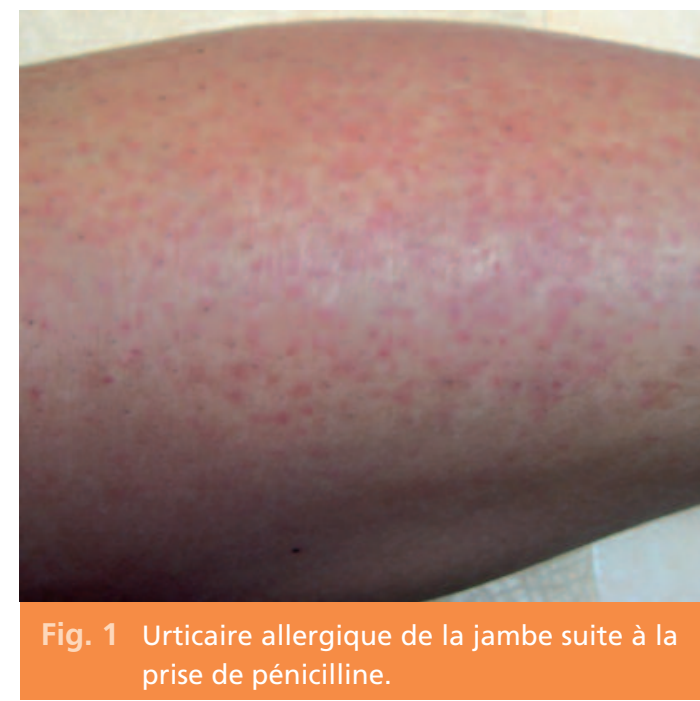

Dans ces situations, il est important de définir la molécule allergène car cela peut aussi être une éventuelle allergie au latex des gants du praticien ou encore à l'iode de la bétadine... Le patient sera donc adressé chez un allergologue afin d'éclaircir la situation et d'éviter ainsi d'éventuels désagréments lors des soins.

\section{Les pathologies générales et les médications en cours nécessitant des précautions [2]}

(Voir tableau I).

\section{> Choisir sa molécule anesthésique}

Deux molécules sont à notre disposition :

- molécule anesthésique à fonction amide : métabolisée par le foie. Il faut donc éviter de l'utiliser chez les patients souffrant de pathologies hépatiques (cirrhose, hépatites virales...) ;

- molécule anesthésique à fonction ester : éliminée dans le plasma.

$>$ Choisir avec ou sans vasoconstricteur et le type de vasoconstricteur

- Avec vasoconstricteur : très apprécié en odontologie, il diminue la résorption systémique des molécules anesthésiques, augmente leur durée d'action et assure une hémostase locale efficace.

Cependant, il doit être utilisé avec parcimonie : ne pas dépasser 2 cartouches à 1/100 000 ou 4 cartouches à 1/200 000 chez les patients souffrant de troubles cardiovasculaires et traités par des bêtabloquants.

Deux types de vasoconstricteur sont à notre disposition, l'épinéphrine et la norépinéphrine. L'épinéphrine est préférée à la norépinéphrine sauf chez les patients allergiques ou diabétiques insulinodépendants du fait du caractère hyperglycémiant de l'épinéphrine.

- Sans vasoconstricteur : en cas de contre-indication [3].

Les pathologies qui contre-indiquent formellement l'utilisation de vasoconstricteurs sont :

- le phéochromocytome, tumeur rare, le plus souvent bénigne, située au niveau des médullosurrénales et de la chaîne sympathique ;

- I'os irradié au-delà de 40 Gy, celui-ci étant moins bien vascularisé ;

- I'arythmie cardiaque ;

- l'infarctus datant de moins de 6 mois ;

- l'allergie aux sulfites.

En revanche, certains états physiologiques permettent tout à fait I'utilisation des vasoconstricteurs :

- la grossesse et l'allaitement, les doses usuelles peuvent être utilisées ;

- l'enfant au-delà de six mois, seule la dose totale d'anesthésique local doit être adaptée au poids de l'enfant qu'il y ait ou non vasoconstricteur ;

- chez la personne âgée, la dose totale d'anesthésique avec ou sans vasoconstricteur doit être adaptée à l'état métabolique du sujet considéré (insuffisance rénale ou hépatique). 


\section{La technique anesthésique}

En cas de problème d'hémostase lié à un traitement anticoagulant ou à une maladie de
I'hémostase, toute anesthésie tronculaire est contre-indiquée. L'anesthésie générale (AG) en milieu hospitalier est ainsi parfois une solution à envisager.

\section{Les troubles de l'hémostase : existe-t-il un risque hémorragique?}

\section{Qu'est-ce que l'hémostase?}

L'hémostase est une réaction de défense de l'organisme face à une hémorragie en activité ou imminente [4] (fig. 2).

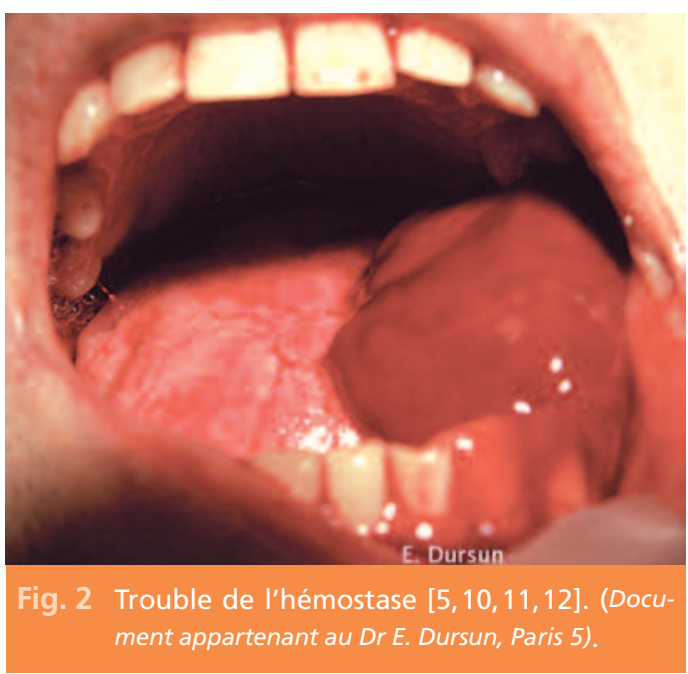

Classiquement, l'hémostase est divisée en trois composantes [5] :

- I'hémostase primaire qui aboutit à la formation du clou plaquettaire dont la fonction est de colmater la brèche vasculaire ;

- la coagulation qui donne naissance au caillot de fibrine, lequel assure la stabilité et la durabilité du clou plaquettaire pendant toute la période de guérison du tissu lésé ;

- la fibrinolyse qui permet d'éliminer le caillot fibrino-plaquettaire pour rétablir la circulation sanguine.

\section{Les questions \\ à poser au patient}

Le praticien doit faire préciser par le patient:

- le passé des saignements anormaux, apparus depuis la petite enfance (chute d'une dent

Tableau III Caractéristiques des troubles de l'hémostase primaire et de la coagulation [13].

\begin{tabular}{l|l}
\multicolumn{1}{c|}{ Trouble de l'hémostase primaire } & \multicolumn{1}{c}{ Trouble de la coagulation } \\
\hline Hémorragie est : & Hémorragie est : \\
- spontanée & $\begin{array}{l}\text { - provoquée par un traumatisme minime } \\
\text { - unique }\end{array}$ \\
- nombreuse & - localisé aux niveaux des tissus profonds (hématome) \\
- localisée au niveau de la peau et des muqueuses & - de volume moyen ou important (litre) \\
(pétéchies et ecchymoses) & - différée \\
- de petit volume (ml) & - récidivante \\
- immédiate & - pouvant perturber la cicatrisation \\
- cesse après une compression locale de 15 min & \\
- ne récidive pas & \\
- ne perturbe pas la cicatrisation &
\end{tabular}


temporaire...) ou plus tard dans la vie d'adulte (extraction, épistaxis, gingivorragies sans cause apparente, menstruation abondante...) ;

- la survenue du saignement : spontané, répété sans cause traumatique déclenchante objectivable ou provoqué par un traumatisme léger qui normalement n'entraîne pas d'hémorragie ;

- la localisation du saignement: diffuse, provenant de plusieurs territoires cutanés, ou localisée et dans ce cas plus évocatrice d'une pathologie ;

- le caractère récidivant : après les quelques heures ou quelques jours suivant le traumatisme et dans quelles circonstances ?

- la nécessité d'une transfusion sanguine après un acte chirurgical courant (extraction) et son explication ;

- l'existence d'un syndrome hémorragique chez les membres d'une même famille avec souvent la présence de mariage consanguin ;

- l'apparition d'ecchymoses fréquentes sans cause apparente ;

- les pathologies générales ou les médications pouvant interférer avec l'hémostase : notamment les patients souffrant de pathologies cardiovasculaires et qui suivent un traitement anticoagulant (antiagrégant plaquettaire [AAP] et antivitamine $K$ [AVK]) ou des patients présentant des problèmes hépatiques.

\section{Un trouble génétique \\ ou acquis?}

Au terme du questionnaire, le praticien doit savoir si le patient souffre de trouble de l'hémostase et si ce trouble est génétique ou acquis. Les troubles génétiques sont rares et précoces dans la vie de l'individu, spécifiques à un défaut connu de I'hémostase (maladie de Willebrand, hémophilies $A$ et $B$...).
Les troubles acquis sont en relation soit avec une pathologie (maladie hépatique, leucémie...), soit avec un traitement (chimiothérapie, radiothérapie) soit avec une médication (à base d'antiagrégant plaquettaire de type aspirine ou d'anticoagulant oraux type AVK (Préviscan ${ }^{\circledR}$ ).

De même, le praticien peut savoir si le trouble de I'hémostase se situe au niveau de l'hémostase primaire ou de la coagulation (tableau III).

\section{Quelle attitude thérapeutique}

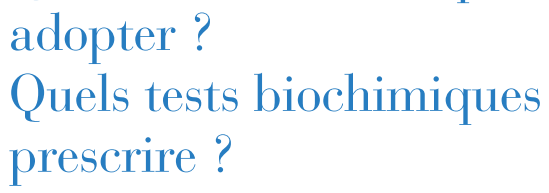

L'attitude thérapeutique appropriée est intimement dépendante des informations cliniques obtenues. Le praticien pourra en plus :

- rechercher au cours de l'examen clinique les manifestations révélatrices du trouble suspecté (ecchymose, pétéchie) ;

- prescrire les tests biochimiques de première ligne : numération plaquettaire (NP) et temps de saignement (TS) pour analyser l'hémostase primaire ; temps de Quick (TQ), International Normalized Ratio (INR), temps de céphaline activée (TCA) pour la coagulation.

La prescription de ces tests, qui dépend du syndrome hémorragique, est soit orientée, soit systématique en situation préopératoire. En cas de pathologie connue, le patient est souvent suivi par un hématologue ou un cardiologue avec qui on doit prendre contact. Un INR est nécessaire avant toute extraction chez un patient sous AVK. Quel que soit le trouble de l'hémostase, si le patient peut être soigné en ville, il faut prévoir tous les moyens d'hémostase locale mis à disposition pour juguler I'hémorragie attendue (antifibrinolytique, colle hémostatique, sutures, compression prolongée à l'aide d'une gouttière ou d'une prothèse amovible....). 


\section{$>$ Modalité de prise en charge \\ d'un patient sous AAP \\ en chirurgie bucco-dentaire [6]}

Ainsi lorsqu'un patient est sous AAP, il faut évaluer le risque médical qu'il présente et rechercher les facteurs qui peuvent potentialiser le saignement. Mais aucun examen biologique n'est actuellement suffisamment performant pour prédire avec certitude le risque hémorragique encouru.

L'évaluation du risque de saignement repose donc essentiellement sur l'interrogatoire médical et l'examen clinique.

Ces patients peuvent la plupart du temps être soignés en cabinet de ville en respectant une technique d'anesthésie et une hémostase chirurgicale rigoureuse avec sutures des berges de la plaie opératoire et compression locale, ainsi que le recours à des hémostatiques locaux résorbables.

\section{> Modalités de prise en charge d'un patient traité par AVK en chirurgie bucco-dentaire [7]}

Un contact préalable par écrit avec le médecin responsable du suivi du traitement par AVK du patient est indispensable et permettra d'orienter le type de prise en charge hospitalière ou en cabinet de ville.

La prise en charge de ce type de patients peut se faire en pratique de ville par des praticiens disposant du plateau technique nécessaire (moyens d'hémostase locale notamment), pour des patients dont I'INR est inférieur ou égal à 3 et

\section{Anxiété et stress}

Dès l'établissement de l'EM, le praticien doit juger de l'état de stress dans lequel se trouve le patient et évaluer si son anxiété peut devenir pathologique et invalidante. Dans ce cas, les pour des actes sans risque hémorragique ou à risque hémorragique modéré ; par exemple pour des avulsions en secteur localisé ou la mise en place d'un implant unitaire.

Une prise en charge hospitalière est recommandée si l'INR est supérieur à 3 et/ou si le risque hémorragique est élevé et/ou s'il existe un risque médical associé (notamment un patient traité par l'association AVK/AAP).

L'instauration d'un relais du traitement par AVK à l'aide d'héparine de bas poids moléculaire (HBPM) ou d'héparine non fractionnée (HNF) en milieu hospitalier avant, pendant et après la phase chirurgicale est possible mais doit rester exceptionnelle. Seul le médecin est habilité à définir le protocole approprié à la situation clinique.

Les anesthésies locorégionales sont déconseillées. L'anesthésique local doit contenir un vasoconstricteur sauf dans les rares cas de contre-indication de son emploi (arythmie non stabilisée, les patients présentant un phéochromocytome...).

Dans les cas d'avulsions dentaires, la mise en place d'un matériau hémostatique résorbable intra-alvéolaire doit être systématique. Toute plaie intrabuccale doit être suturée. Les fils de suture peuvent être résorbables ou non. Les points doivent être unitaires. Dans les cas d'interventions chirurgicales hémorragiques, l'utilisation de colle biologique et/ou d'agent antifibrinolytique (Exacyl ${ }^{\circledR}$ ampoule suspension buvable) est recommandée. Une compression locale doit être mise en place immédiatement en postopératoire pendant au moins 10 minutes.

facultés d'adaptation sont dépassées et différentes manifestations cliniques peuvent être observées allant du malaise psychique aux troubles cardiaques et respiratoires. 


\section{Les questions à poser}

L'entretien oral permet de préciser :

- les conditions dans lesquelles les soins chirurgicaux antérieurs se sont déroulés et si le patient a été victime d'une crise d'angoisse (peur de la douleur, du caractère invasif de l'acte...) ;

- si le patient est psychologiquement fragile, traité pour une dépression nerveuse ou pour des problèmes psychiatriques ;

- s'il est surmené pour des raisons professionnelles (licenciement, examen...) ou privées (divorce, perte d'un être cher...) ;

- s'il existe une maladie organique liée au stress: ulcère gastroduodénal, rectocolite hémorragique, hypertension artérielle, maladie coronarienne, asthme, certaines maladies immunitaires, maladies cutanées (allergie, psoriasis, urticaire, eczéma), si le patient suit une thérapie à base de corticoïdes.

Les corticoïdes inhibent la production endogène de stéroïdes et provoquent une intolérance au stress. En fonction des informations fournies par I'EM, le praticien peut identifier les prédispositions au stress et orienter son attitude thérapeutique.

\section{L'attitude thérapeutique à adopter}

En cas de dépression, le contact peut s'avérer difficile car le patient n'est pas toujours capable de percevoir correctement nos informations. De même, il est plus prudent en présence de maladies liées au stress ou de patient sous corticothérapie d'effectuer des actes de courte durée avec une anesthésie efficace, de contacter le médecin traitant si le traitement en cours doit être modifié (augmentation de la dose des corticoïdes) et de prescrire si nécessaire une prémédication sédative en demandant au patient de venir accompagné par une tierce personne.

La sédation consciente par inhalation du mélange équimolaire d'oxygène et du protoxyde d'azote (MEOPA) constitue en odontologie une excellente solution pour réduire I'anxiété et améliorer la coopération, permettant ainsi la réalisation des soins dentaires dans de meilleures conditions. Sa facilité d'administration, sa rapidité d'induction et d'élimination et le peu d'effets indésirables qu'il engendre font du MEOPA un agent sédatif de choix. La sédation consciente se révèle, de surcroît, être une alternative efficace à l'anesthésie générale chez les enfants peu coopérants, anxieux ou mentalement déficients devant bénéficier de soins dentaires ou chez des adultes handicapés moteurs. La formation certifiée du praticien à ces techniques est indispensable.

\section{Tableau IV Risque A et Risque B [14].}

\begin{tabular}{|c|c|}
\hline $\begin{aligned} \text { Risque } A= & \text { risque de surinfection identifié localement } \\
& \text { et/ou surinfection générale }\end{aligned}$ & $\begin{aligned} \text { Risque } B= & \text { risque de surinfection lié à une localisation } \\
& \text { secondaire de la bactérie à l'origine d'un } \\
& \text { nouveau foyer infectieux situé à distance } \\
& \text { du lieu de l'acte dentaire réalisé }\end{aligned}$ \\
\hline $\begin{array}{l}\text { - Sujets transplantés ou greffés sous thérapeutique immuno- } \\
\text { suppressive }\end{array}$ & $\begin{array}{l}\text { - Patients ayant des prothèses d'organe autre que valvu- } \\
\text { laires }\end{array}$ \\
\hline $\begin{array}{l}\text { - Sujets immunodéprimés d'origine congénitale ou médica- } \\
\text { menteuse }\end{array}$ & - Patients à risque d'endocardite infectieuse (cf. tableau V) \\
\hline $\begin{array}{l}\text { - Pathologies associées non contrôlées (diabète, insuffisance } \\
\text { rénale et/ou hépatique, sujets ayant des infections chro- } \\
\text { niques) }\end{array}$ & \\
\hline - Sujets dénutris & \\
\hline
\end{tabular}


Toutes ces précautions limitent le risque d'aggravation de la maladie. Toutefois il est préférable de reporter le rendez-vous, notamment pour les actes chirurgicaux, si le stress du patient ne peut être maîtrisé.

\section{Antibioprophylaxie, antibiothérapie : existe-il un risque infectieux?}

\section{L'antibiothérapie prophylactique}

Une EM rigoureuse est essentielle pour dépister les patients susceptibles aux infections et dont l'état de santé impose une antibiothérapie prophylactique avant la réalisation d'actes qualifiés « à risque ». Toute effraction vasculaire est considérée comme une porte d'entrée pour les micro-organismes (tableau VIII).

Les indications d'une antibiothérapie prophylactique sont répertoriées dans les tableaux IV, V, VI, VII.

Toute effraction vasculaire nécessite une antibiothérapie prophylactique chez les sujets à risque $A$ et $B$. (Risque $A=$ risque de surinfection identifié localement et/ou surinfection générale. Risque $B=$ risque de surinfection lié à une localisation secondaire de la bactérie à l'origine d'un nouveau foyer infectieux situé à distance du lieu de l'acte dentaire réalisé.)

\section{> Patients à risque $A$}

Six situations couramment rencontrées de nos jours entrent dans la catégorie des patients $A$.

- Le diabète instable et/ou de longue durée : en accord avec le médecin traitant, une antibiothérapie prophylactique est indiquée pour éviter les problèmes de cicatrisation et d'infection postopératoire. L'EM nous renseigne sur la date de début de la maladie et celle du traitement ainsi que les médications suivies en cours. La connaissance du taux d'hémoglobine glyquée ( $\mathrm{Hb} 1 \mathrm{Ac}$ ) est nécessaire pour connaître la sévérité de l'instabilité.

- Les patients immunodéprimés ou traités par corticothérapie au long cours doivent être également traités par antibiothérapie prophylactique avant toute intervention chirurgicale.

- Les patients en chimiothérapie : une étroite collaboration avec l'oncologue médical est

Tableau V Cardiopathies à risque d'endocardite infectieuse [14]

\begin{tabular}{l|l}
\multicolumn{1}{c|}{ Cardiopathies à haut risque } & \multicolumn{1}{c}{ Cardiopathies à risque modéré } \\
\hline - Prothèses valvulaires & - Autres cardiopathies congénitales (cardiopathies non \\
- Antécédents d'endocardite infectieuse & cyanogènes sauf communication interauriculaire) \\
- Cardiopathies congénitales cyanogènes & - Valvulopathies : insuffisance, rétrécissement, et bicuspi- \\
- Dérivations chirurgicales (pulmonaires-systémiques) & die aortiques, insuffisance mitrale \\
& - Dysfonctions valvulaires acquises \\
& - Prolapsus de la valve mitrale avec insuffisance mitrale \\
& et/ou épaississement valvulaire \\
& - Cardiomyopathie hypertrophique obstructive
\end{tabular}


Tableau VI Indication de l'antibioprophylaxie au cours des actes bucco-dentaires invasifs chez le sujet sain et chez le sujet à risque A ou B [14].

\begin{tabular}{|c|c|c|c|c|c|}
\hline \multirow[b]{2}{*}{$\begin{array}{l}\text { ACTES BUCCO-DENTAIRES INVASIFS } \\
\text { (avec risques de saignement significatif) }\end{array}$} & \multicolumn{2}{|c|}{ SUJET SAIN } & \multicolumn{3}{|c|}{ SUJET À RISQUE } \\
\hline & $\begin{array}{c}\text { Risque } \\
\text { d'infection }\end{array}$ & $\begin{array}{c}\text { Antibio- } \\
\text { prophylaxie }\end{array}$ & $\begin{array}{c}\text { Risque } \\
\text { d'infection }\end{array}$ & $\begin{array}{l}\text { Antibiopr } \\
\text { chez le } \\
\text { à risque d } \\
\text { locale / } \\
\text { générale } \\
\text { Risque } \mathrm{A}\end{array}$ & $\begin{array}{l}\text { ophylaxie } \\
\text { e sujet } \\
\text { l'infection } \\
\text { à distance } \\
\text { Risque B }\end{array}$ \\
\hline Anesthésies locales intraligamentaires & Non & NJ & & ND & R \\
\hline Mise en place d'une digue & Non & NJ & & NJ* & $\mathrm{R}$ \\
\hline $\begin{array}{l}\text { Soins endodontiques } \\
\text { - Traitements des dents à pulpe vitale } \\
\text { - Traitement des dents à pulpe non vitale } \\
\text { y compris la reprise de traitement canalaire }\end{array}$ & $\begin{array}{l}\text { Non } \\
\text { Oui }\end{array}$ & NJ & & $\begin{array}{l}\mathrm{R} \\
\mathrm{R}\end{array}$ & Ro \\
\hline Soins prothétiques à risque de saignement & Non & NJ & & $\mathrm{R}$ & $\mathrm{R}$ \\
\hline $\begin{array}{l}\text { Soins parodontaux non chirurgicaux } \\
\text { - Détartrage avec et sans surfaçage } \\
\text { - Sondage }\end{array}$ & $\begin{array}{l}\text { Non } \\
\text { Non }\end{array}$ & $\begin{array}{l}\text { NJ } \\
\text { NJ }\end{array}$ & & $\begin{array}{l}\text { ND } \\
\text { ND }\end{array}$ & $\begin{array}{l}\mathrm{R} \\
\mathrm{R}\end{array}$ \\
\hline $\begin{array}{l}\text { Actes chirurgicaux } \\
\text { - Avulsions dentaires } \\
\text { - Dent saine } \\
\text { - Dent infectée } \\
\text { - Alvéolectomie } \\
\text { - Séparation de racines } \\
\text { - Amputation radiculaire } \\
\text { - Dent incluse } \\
\text { - Dent en désinclusion } \\
\text { - Germectomie }\end{array}$ & $\begin{array}{l}\text { Non } \\
\text { Oui } \\
\text { Non } \\
\text { Non } \\
\text { Non } \\
\text { Oui } \\
\text { Oui } \\
\text { Oui }\end{array}$ & $\begin{array}{l}\text { NJ } \\
\text { ND } \\
\text { NJ } \\
\text { NJ } \\
\text { NJ } \\
\text { ND } \\
\text { R } \\
\text { ND }\end{array}$ & & $\begin{array}{l}R \\
R \\
R \\
R \\
R \\
R \\
R \\
R\end{array}$ & $\begin{array}{c}R \\
R \\
R \\
R \\
\text { SO } \\
R \\
R \\
R\end{array}$ \\
\hline - Transplantation / Réimplantations & Oui & $\mathrm{R}$ & & $\mathrm{R}$ & SO \\
\hline - Chirurgie péri-apicale & Oui & $\mathrm{R}$ & OUI & $\mathrm{R}$ & so \\
\hline $\begin{array}{l}\text { - Chirurgie des tumeurs bénignes de la cavité buccale } \\
\text { - Maxillaires (kyste...) } \\
\text { - Tissus mous }\end{array}$ & $\begin{array}{l}\text { Oui } \\
\text { Non }\end{array}$ & $\begin{array}{l}\text { R } \\
\text { NJ }\end{array}$ & & $\begin{array}{l}\mathrm{R} \\
\mathrm{R}\end{array}$ & $\begin{array}{l}R \\
R\end{array}$ \\
\hline $\begin{array}{l}\text { - Chirurgie parodontale } \\
\text { - Chirurgie de la poche } \\
\text { - Lambeau d'accès } \\
\text { - Comblement et greffes osseuses } \\
\text { - Membranes } \\
\text { - Chirurgie mucogingivale } \\
\text { - Lambeau } \\
\text { - Greffes }\end{array}$ & $\begin{array}{l}\text { Non } \\
\text { Oui } \\
\text { Oui } \\
\text { Non } \\
\text { Non }\end{array}$ & $\begin{array}{l}\text { NJ } \\
R \\
R \\
\text { NJ } \\
\text { NJ }\end{array}$ & & $\begin{array}{l}R \\
R \\
R \\
R \\
R\end{array}$ & $\begin{array}{l}\text { So } \\
\text { so } \\
\text { so } \\
\text { so } \\
\text { so }\end{array}$ \\
\hline - Freinectomies & Non & NJ & & R & $\mathrm{R}$ \\
\hline - Biopsie des glandes salivaires accessoires & Non & NJ & & $\mathrm{R}$ & $\mathrm{R}$ \\
\hline - Chirurgie osseuse & Oui & $\mathrm{R}$ & & $\mathrm{R}$ & $\mathrm{R}$ \\
\hline $\begin{array}{l}\text { - Chirurgie implantaire } \\
\text { - Mise en place } \\
\text { - Dégagement (stade II) }\end{array}$ & $\begin{array}{l}\text { Oui } \\
\text { Non }\end{array}$ & $\begin{array}{l}\text { R } \\
\text { NJ }\end{array}$ & & $\begin{array}{l}R \\
R\end{array}$ & $\begin{array}{l}\text { SO } \\
\text { SO }\end{array}$ \\
\hline - Mise en place de matériaux de comblement & Oui & $\mathrm{R}$ & & $\mathrm{R}$ & SO \\
\hline Orthopédie dento-faciale & & & & & \\
\hline $\begin{array}{l}\text { - Traitement en orthopédie dento-faciale } \\
\text { (mise en place de bagues orthodontiques) }\end{array}$ & Non & NJ & & $\mathrm{R}$ & so \\
\hline $\begin{array}{l}\text { - Chirurgie préorthodontique des dents incluses } \\
\text { ou enclavées }\end{array}$ & Non & NJ & & $\mathrm{R}$ & so \\
\hline
\end{tabular}

$\mathrm{NJ}=$ non justifiée $; \mathrm{ND}=$ non déterminée : sans preuves scientifiques, études à prévoir ; $\mathrm{R}=$ recommandée par accord professionnel ;

SO = sans objet : patients concernés par l'indication antibioprophylaxie (acte contre-indiqué chez ce type de sujet) ; * acte non à risque chez ce type de sujet. 
nécessaire pour permettre de planifier et d'optimiser au mieux les soins avant et entre deux cures.

- Avant :

- suppression des foyers infectieux et suppressions des facteurs d'irritation mécanique. Le fait de pouvoir intervenir avant la chimiothérapie permet d'être plus conservateur (traitement endodontique, traitement parodontal) et d'éviter le recours aux extractions en cas d'infections per- et post-chimiothérapie.

\section{- Pendant :}

- il faut tenir compte de la situation hématologique du patient, si un acte est à réaliser en urgence, un bilan hématologique (NFS et bilan d'hémostase) doit être prescrit pour déterminer les conditions optimales de soins. Si les leucocytes sont $<2000 / \mathrm{mm}^{3}$ et plaquettes $<100000 / \mathrm{mm}^{3}$, il est préférable que le patient soit hospitalisé ;

- il faut insister sur l'importance des manœuvres d'hygiène du patient (brosse à dents souple, bain de bouche antiseptique, remise en état de la denture).

\section{- Les patients sous biphosphonates (BP) [8]}

Administrés par voie intraveineuse (IV), les BP sont indiqués dans la prise en charge des myélomes multiples, la prévention des complications osseuses de certaines tumeurs malignes avancées, et le traitement des hypercalcémies malignes (fig. $\mathbf{3}$ ).

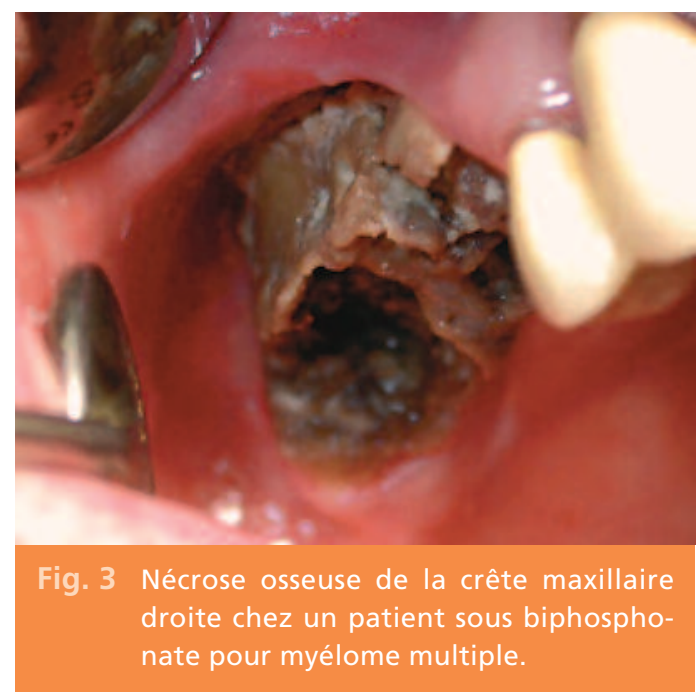

Tableau VII Traitements prophylactiques lors de certains actes dentaires [14].

\begin{tabular}{|c|c|c|}
\hline Situation & Antibiotique & Contre-indications \\
\hline Prophylaxie standard & Amoxicilline & $\begin{array}{l}\text { Adulte : } 3 \mathrm{~g}^{*} \\
\text { Enfant : } 50 \mathrm{mg} \cdot \mathrm{kg}^{-1} \\
\text { per os } 1 \mathrm{~h} \text { avant le geste }\end{array}$ \\
\hline \multirow[t]{2}{*}{ Allergie aux $\beta$-lactamines } & Clindamycine & $\begin{array}{l}\text { Adulte : } 600 \mathrm{~g} \\
\text { Enfant : } 15 \mathrm{mg} \cdot \mathrm{kg}^{-1} \\
\text { per os } 1 \mathrm{~h} \text { avant le geste }\end{array}$ \\
\hline & Pristinamycine & $\begin{array}{l}\text { Adulte }: 1 \mathrm{~g} \\
\text { Enfant : } 25 \mathrm{mg} \cdot \mathrm{kg}^{-1} \\
\text { per os } 1 \mathrm{~h} \text { avant le geste }\end{array}$ \\
\hline Voie orale inutilisable & Amoxicilline & $\begin{array}{l}\text { Adulte : } 2 \mathrm{~g} \text { IV (perfusion de } 30 \mathrm{mn} \text { ) dans l'heure précédant le geste, } \\
\text { puis } 1 \mathrm{~g} \text { per os } 6 \mathrm{~h} \text { plus tard } \\
\text { Enfant : } 50 \mathrm{mg} \cdot \mathrm{kg}^{-1} \mathrm{IV} \text { (perfusion de } 30 \mathrm{mn} \text { ) dans I'heure précédant le } \\
\text { geste, puis } 25 \mathrm{mg} \cdot \mathrm{kg}^{-1} \text { per os } 6 \mathrm{~h} \text { plus tard }\end{array}$ \\
\hline \multirow[t]{2}{*}{$\begin{array}{l}\text { Allergie aux } \beta \text {-lactamines } \\
\text { et voie orale inutilisable }\end{array}$} & Vancomycine** & $\begin{array}{l}\text { Adulte : } 1 \mathrm{~g} \mathrm{IV} \mathrm{(perfusion} \mathrm{de} 60 \mathrm{mn} \text { ) dans l'heure précédant le geste } \\
\text { Enfant : } 20 \mathrm{mg} \cdot \mathrm{kg}^{-1} \mathrm{IV} \text { (maximum } 1 \mathrm{~g} \text { ) dans I'heure précédant le geste }\end{array}$ \\
\hline & Teicoplanine & $\begin{array}{l}\text { Adulte : } 400 \mathrm{~g} \text { IV (directe) dans l'heure précédant le geste } \\
\text { Enfant : pas encore d'AMM en prophylaxie }\end{array}$ \\
\hline
\end{tabular}


Administrés par voie orale à faible dose, les BP sont indiqués dans le cas d'ostéoporose post-ménopausique chez les femmes à haut risque de fracture, le traitement de l'ostéoporose masculine, de l'ostéoporose corticoinduite.

Le praticien odontologiste peut être confronté à deux situations cliniques différentes:
- Le patient consulte avant la prise du traitement.

- par IV : faire un bilan radiographique complet pour éliminer tous les foyers infectieux et attendre la cicatrisation muqueuse et osseuse si possible avant de commencer le traitement (soit quatre mois) ;

- par voie orale : faire le bilan radiographique et les soins nécessaires. Ces soins ne

\section{Tableau VIII Indication de l'antibiothérapie dans les infections dentaires et du parodonte chez} le sujet sain et le sujet à risque d'infection A ou B [14].

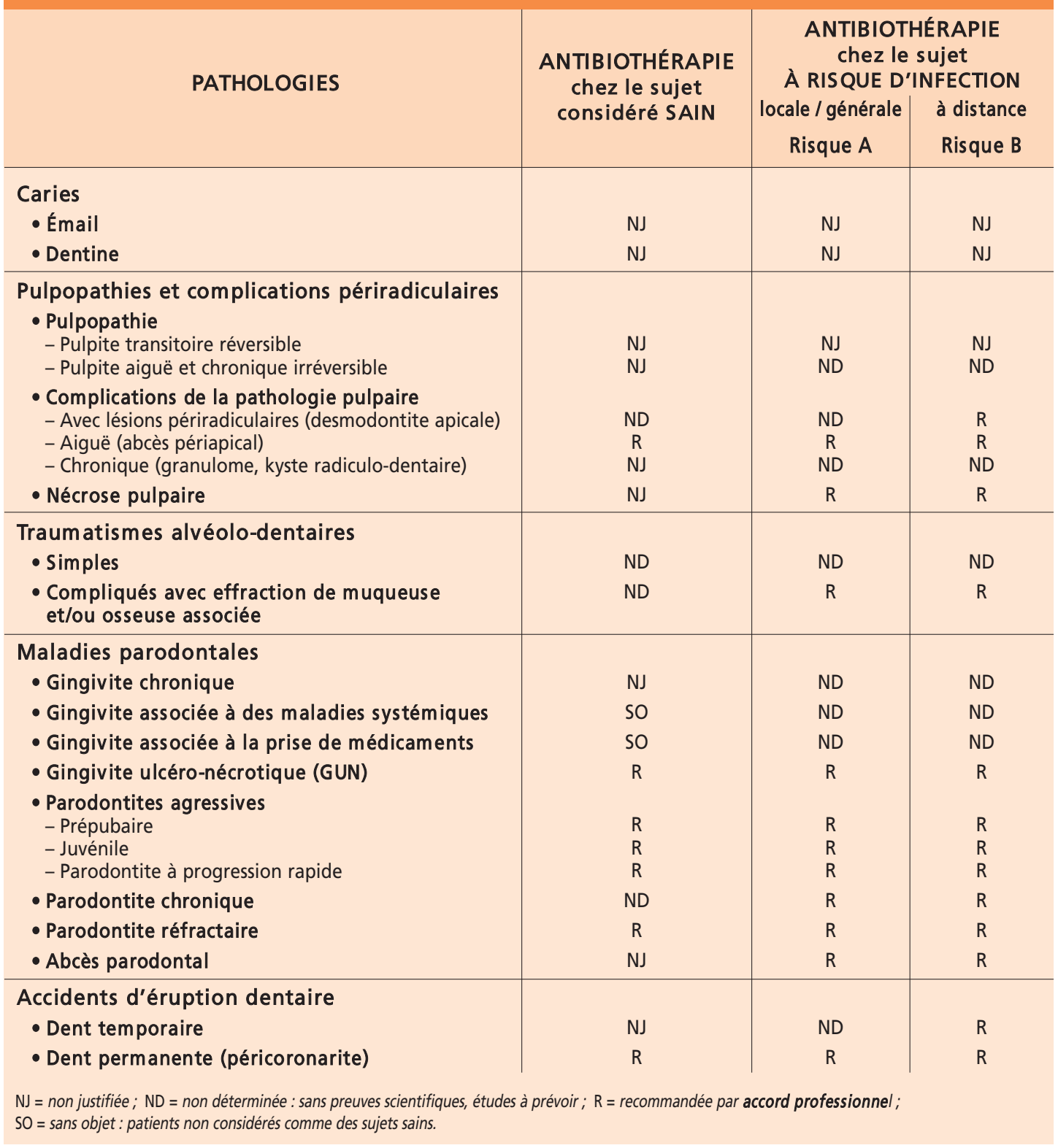


doivent pas retarder l'instauration du traitement par BP chez les patients à risque élevé de fractures.

- Le patient consulte en cours de traitement ou a déjà été traité.

- par IV :

- réaliser un suivi : celui-ci sera pratiqué par un spécialiste tous les quatre mois et au moindre symptôme bucco-dentaire, en collaboration avec l'oncologue ;

- dépister et traiter les foyers infectieux par des gestes aussi peu agressifs que possible pour l'os, le parodonte et la muqueuse. II faut éviter d'intervenir mais, en cas d'obligation (mobilité dentaire $>3$ ou foyer infectieux avéré), une antibiothérapie (à commencer la veille et jusqu'à la fin de la cicatrisation) et un protocole strict (utilisation d'anesthésique sans vasoconstric- teur, éviter de traumatiser l'os et de laisser de l'os exposé dans la cavité buccale en réalisant des sutures hermétiques) sont indispensables ;

- par voie orale : réaliser un suivi bucco-dentaire au minimum une fois par an.

\section{Attention :}

- le patient doit être averti du risque d'ostéonécrose, de la nécessité d'avoir une hygiène efficace et de réaliser des contrôles réguliers par son praticien ;

- le patient doit être averti qu'en cas de douleur, d'inflammation ou d'infections buccales (le patient constate : rougeur, gonflement, température), il doit venir consulter en urgence.

Si l'ostéonécrose est avérée, le patient doit être adressé en milieu hospitalier.

Tableau IX Indication de I'antibiothérapie dans les autres infections bucco-dentaires chez le sujet sain et le sujet à risque d'infection A ou B [14].

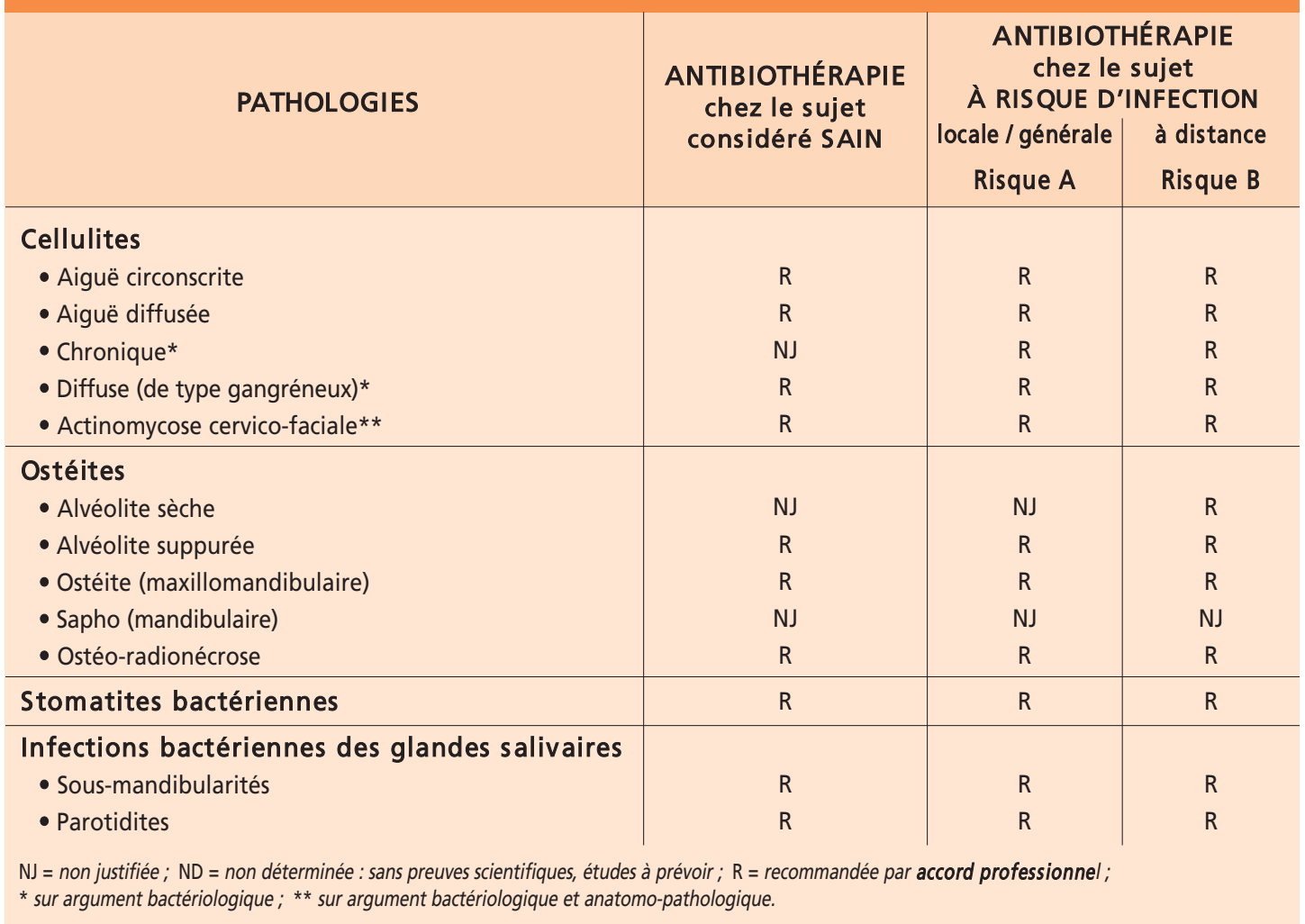




\section{- Le patient irradié au niveau orofacial [9]}

La prise en charge bucco-dentaire avant, pendant et après la radiothérapie est indispensable quelles que soient les modalités de I'irradiation. Elle doit être adaptée selon le territoire irradié.

Les complications bucco-dentaires peuvent être prévenues par une mise en état préalable de la denture (éradication des foyers infectieux ou des dents suspectes).

Les caries dentaires souvent consécutives à I'hyposialie peuvent être évitées par une fluorothérapie.

L'avulsion d'une dent dans la zone d'irradiation peut être à l'origine d'une ostéoradionécrose. II est nécessaire de pratiquer cet acte sans utiliser de vasoconstricteur, avec une asepsie rigoureuse sous couvert d'une antibiothérapie et après un bilan hématologique. La suture des berges est souhaitable ainsi que I'utilisation de colle biologique (disponible uniquement en milieu hospitalier).

\section{- Le patient dénutri alcoolo-tabagique}

Chez le patient alcoolo-tabagique qui présente des problèmes psychologiques associés à une hygiène bucco-dentaire défectueuse et à des troubles hématologiques, la chirurgie parodontale est contre-indiquée.

\section{$>$ Patients à risque $B$}

Les cardiopathies qui prédisposent au développement d'une endocardite infectieuse (tableaux IV et V) font l'objet des mêmes actes contre-indiqués et des mêmes recommandations que pour les patients à risque d'endocardites.

En ce qui concerne les infections sur prothèses articulaires (tableau IV), en I'absence de recommandations spécifiques, la prise en charge de ces patients s'apparente à celle réalisée chez les patients à risque d'endocardite.

\section{L'antibiothérapie curative}

Elle concerne les infections bucco-dentaires bactériennes. Elles sont classées selon leurs

\section{Tableau X Indication de l'antibiothérapie dans la pathologie des implants et la régénération tissulaire parodontale chez le sujet sain et le sujet à risque d'infection A ou B [14].}

\begin{tabular}{|c|c|c|c|}
\hline \multirow[t]{2}{*}{ PATHOLOGIES } & \multirow{2}{*}{$\begin{array}{l}\text { ANTIBIOTHÉRAPIE } \\
\text { chez le sujet } \\
\text { considéré SAIN }\end{array}$} & \multicolumn{2}{|c|}{$\begin{array}{c}\text { ANTIBIOTHÉRAPIE } \\
\text { chez le sujet } \\
\text { À RISQUE D'INFECTION }\end{array}$} \\
\hline & & $\begin{array}{c}\text { locale / générale } \\
\text { Risque } A\end{array}$ & $\begin{array}{c}\text { à distance } \\
\text { Risque B }\end{array}$ \\
\hline \multicolumn{4}{|l|}{ Pathologies associées aux implants dentaires } \\
\hline \multicolumn{4}{|l|}{ Lésions péri-implantaires infectieuses ("péri-implantites») } \\
\hline $\begin{array}{l}\text { - Précoces, avant mise en charge de l'implant dentaire } \\
\text { (4 à } 6 \text { mois post-opératoire) }\end{array}$ & NJ & $\mathrm{R}$ & so \\
\hline $\begin{array}{l}\text { - Tardives, après mise en charge de l'implant dentaire } \\
\text { (>4 à } 6 \text { mois) }\end{array}$ & NJ & R & so \\
\hline \multicolumn{4}{|l|}{ Régénération tissulaire parodontale } \\
\hline • Membranes (intérêt ?) & ND & $\mathrm{R}$ & SO \\
\hline - Greffes osseusses & ND & $\mathrm{R}$ & so \\
\hline - Matériaux de complément & ND & $\mathrm{R}$ & SO \\
\hline
\end{tabular}


sites : pulpe et périapex, tissus mous, tissus osseux, parodonte, site d'éruption, sinus maxillaire, glandes salivaires, et infections à

\section{Prescriptions}

Pour éviter les interactions ou les contre-indications médicamenteuses, le praticien doit connaître le traitement exact que suit le patient (type de médicament, posologie, durée du traitement, fréquence), ainsi que les complications qui lui sont associées.

L'automédication est à dépister au sein de l'EM. Par exemple, il convient de vérifier que le patient n'a pas pris d'Aspirine pour maîtriser sa douleur, quelques jours avant ou le jour même du rendez-vous.

L'EM est également très utile pour identifier une pathologie qui risque d'être exacerbée par les médicaments qui sont habituellement prescrits en postopératoire.

Par exemple :

- prudence en cas d'allergie ;

- contre-indications des anti-inflammatoires

\section{Conclusion}

Seule I'EM, lors de l'interrogatoire, permet d'établir l'attitude de l'odontologiste :

- traiter le patient en milieu hospitalier ou en cabinet de ville ;

- utiliser des anesthésiques locaux, avec ou sans vasoconstricteur ;

- pouvoir contrôler l'hémostase ;

- évaluer ou gérer l'anxiété et le stress du patient ;

- prévenir et traiter l'infection ; distance et selon l'état de santé général du patient : sain, risque $A$ ou risque $B$ (tableaux $V$, VI, VII). non stéroïdiens et de l'acide acétylsalicylique en cas d'affection gastro-intestinale ou de trouble de l'hémostase $[6,7]$;

- contre-indication ou posologie adaptée pour les médicaments métabolisés par le foie en cas d'affection hépatique ou néphrotoxique en cas d'insuffisance rénale chronique ;

- changer de molécules en cas de prescriptions multiples antérieures chez un patient à risque d'endocardite [11].

Enfin, I'EM permet de savoir si une patiente est enceinte et donc d'envisager les précautions à prendre en fonction du stade de la grossesse (prescription et contre-indication des médicaments tératogènes) et de prévoir les éventuelles complications liées à cet état physiologique (nausées, diabète, fatigue...) avant toute prescription et intervention.
- orienter la prescription en fonction du
contexte pathologique du patient.

L'avis du médecin traitant reste souvent l'élément primordial de décision. L'odontologiste, quant à lui, éclairé sur le risque encouru, pourra gérer et assurer la prise en charge psychologique du patient, ce dernier étant mieux sensibilisé sur l'importance de son état de santé pour la bonne conduite du traitement bucco-dentaire. 


\section{Bibliographie}

1. Grubwierser GJ, Baubin MA, Strobl HJ, Zangerle RB.

\section{Urgences}

au cabinet dentaire.

Paris : Maloine, 2006.

2. Descroix V, Yasukawa K.

Les médicaments en odonto-stomatologie. Paris : Maloine, 2005.

3. Sites internet : Société française de médecine buccale et chirurgie buccale SFMBCB 2002.

Au sujet de l'emploi des vasoconstricteurs en odonto-stomatologie. http://www.societechirbuc .com/Recommandations/ recommandations vasoconstricteurs.pdf.

4. Jobin $F$.

L'hémostase.

Paris : Maloine, 1995.

5. Dridi SM, Barbey T, Danan M, Wierzba CB. Importance du questionnaire médical en chirurgie buccale et en parodontie. Act Odonto Stomatol 1998,201:15-28.

6. Société française de médecine buccale et chirurgie buccale. Prise en charge des patients sous agents antiplaquettaires

en odontostomatologie.

Recommandations.

http://www.societechirbuc .com/Recommandations/ recommandations_ antiplaquettaires.pdf

7. Société française de médecine buccale et chirurgie buccale.

Prise en charge des patients sous traitement anti-vitamines $\mathrm{K}$ en chirurgie bucco-dentaire. Recommandations. http://www.societechirbuc .com/Recommandations/ recommandations_avk.pdf Consulté en juillet 2009.

8. AFSSAPS

\section{Stratégies}

et recommandations

de I'AFSSAPS

(décembre 2007).

http://www.afssaps.fr/ Infos-de-securite/Lettresaux-professionnels-de-sante/ Recommandations-sur-laprise-en-charge-buccodentaire-des-patients-traitespar-bisphosphonates/ (language)/fre-FR

9. Commission des dispositifs médicaux de l'ADF. Le chirurgien-dentiste face au cancer. dossier ADF, 2009:146-7.
10. Dridi SM, Arreto JD,

Danan M.

Chirurgie et hémostase.

Réalités Cliniques

2000;11:225-38.

11. Ejeil AL, Dridi SM, Wierzba C.

Dépistage des syndromes hémorragiques.

Act Odonto Stomatol 2000;212:467-77.

12. Dridi SM, Arreto $C D$, Gaudy JF, Danan M. Patients traités par anti-vitamines $\mathrm{K}$, que faire en pratique courante? Inf Dent 2005;11:607-14.

13. Aillaud MF. Hémostase et thrombose. 4e édition. Joué-les-Tours : La simarre, 1994.

14. Agence française de sécurité sanitaire des produits de santé. Prescriptions des antibiotiques en odontologie et stomatologie. In : AFSAPPS> dossiers thématiques> antibiothérapie. http://www.afssaps.fr/ Dossiers-thematiques/ Antibiotherapie/ Recommandations/ Consulté en juillet 2009. 


\section{SUMMARY}

\section{Medical set of questions in odontostomatology : necessity, applications, obligations}

Caroline GORIN,

Sophie-Myriam DRIDI, Claude-Bernard WIERZBA, Anne-Laure EJEIL

Keywords

- medical investigation

- medical questionnaire

- recommendations

- risk patients
The medical investigation, often unmarked, is an indispensable tool in our daily practice.

It is in the form of a written questionnaire, dated and signed by the patient completed by an oral interview with the practitioner who can then assess the state general health of the patient, medical monitoring, and medications past, present or future.

The dentist can properly plan the various stages of the treatment plan and take treatment decisions necessary, with all precautions and medical technology necessary.

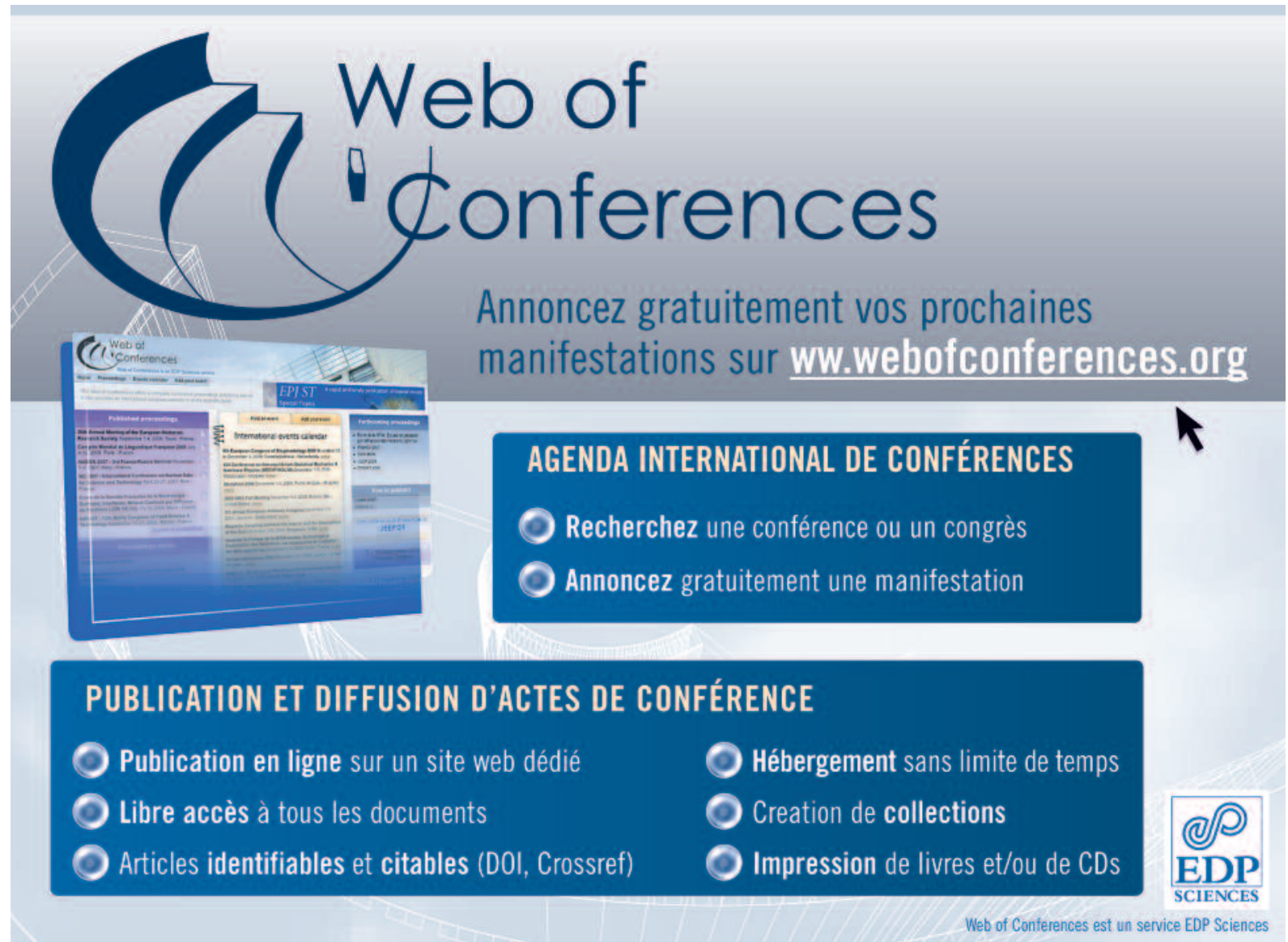

